\title{
Assessing numeracy skills of students entering pharmacy and medicine at one UK University - the potential for interprofessional learning
}

Authors: Sion Coulman, Rob Wilson, Nadia Higgi, Ffion Hawkins, Alex Speakman, Louise Woodgate, Matt Baker and Dai John. Cardiff University

Background: Basic numeracy skills are essential to undergraduate students entering pharmacy and medicine degrees, as there are potential patient safety implications. The aim was to use a contextualised medicines-based numeracy tool to determine the inherent numeracy skills of students entering the first year of pharmacy and medicine programmes at one UK university.

Method: Ethical approval was obtained. A tool consisting of twenty-five calculations to be completed in 45 minutes without a calculator, was administered in October 2011. The tool covered six principal numeracy domains, multiplication, division, percentages, fractions, ratios and unit conversion. On completion candidates provided demographic data and information regarding their mathematical education.

Results: $165 / 168$ pharmacy and 274/284 medicine students sat the diagnostic test in October 2011, producing a completion rate of $97 \%$. Pharmacy students achieved a mean test score of $20.4 / 25$ and medicine students a mean score of 21.3/25. Medical entry students performed better than pharmacy entry students (MannWhitney $U ; p=0.019$ ), with $18 \%$ of medical students achieving full marks compared to $9 \%$ of pharmacy students. Competence was lowest when answering a long multiplication question involving decimals, with 84/168 Pharmacy and 97/284 Medicine students failing to answer the question or answering incorrectly. Twentyone pharmacy $(13 \%)$ and 17 medical $(6 \%)$ students scored 15 or less in the diagnostic test and were thus identified at an early stage as needing additional support, provided by pharmacy and medicine.

Discussion: Although both the pharmacy and medicine faculty were involved in the development, implementation and evaluation of this diagnostic assessment, to date the learning has been uniprofessional. Now that the value of the assessment to both programmes has been established, it may be appropriate to conduct interprofessional sessions for students. There are further opportunities for IPE in medicines-based calculations in later years of the programmes, for example, aligned with medicine's Patient Safety Assessment. 